\title{
Silencing of KNTC1 inhibits hepatocellular carcinoma cells progression via suppressing PI3K/Akt pathway
}

\author{
Hui Tong ${ }^{1, \#}$, Junjie Xie ${ }^{1 \#}$, Xiaohui Liu ${ }^{2}$, Chenghong Peng ${ }^{1}$, Baiyong Shen ${ }^{1, *}$, Zhecheng Zhu ${ }^{1, *}$
} 1Department of General Surgery, Ruijin Hospital, Shanghai Jiao Tong University School of Medicine, Shanghai 200025, China;

2CNRS-LIA124, Sino-French Research Center for Life Sciences and Genomics, State Key Laboratory of Medical Genomics, Rui Jin Hospital, Shanghai Jiao Tong University School of Medicine, Shanghai, 200025, China.

\#These authors contributed equally to this work.

*Correspondence: Zhecheng Zhu; Baiyong Shen, Department of General Surgery, Ruijin Hospital, Shanghai Jiao Tong University School of Medicine, No. 197, Ruijin Second Road, Shanghai 200025, China. Email: zhuzhechengrj@163.com; shenby@shsmu.edu.cn .

\begin{abstract}
Background: Kinetochore associated 1 (KNTC1) encodes a kinetochore component in Rod-Zwilch-ZW10 (RZZ) complex which is essential for the segregation of sister chromatids during mitosis and participates in the spindle checkpoint. Recent research demonstrated that kinetochore proteins may be potential biomarkers and may contribute to the development of human malignancies. Here, we sought to identify the biological significances of KNTC1 in hepatocellular carcinoma (HCC).
\end{abstract}

Methods: KNTC1 expression was studied in HCC tissues by immunohistochemistry. Lentivirus delivered short hairpin RNA (shRNA) was performed to generate KNTC1 knockdown HCC cell lines. The effects of KNTC1 on HCC cells proliferation, migration, apoptosis and tumor formation was analyzed by MTT assay, colony formation assay, wound-healing assay, transwell migration assay, annexin $\mathrm{V}$ assay in vitro and in nude mouse models in vivo.

Results: Our immunohistochemistry experiment showed that KNTC1 was highly expressed in HCC tissues and correlated with terrible prognosis, indicating that KNTC1 acts a pivotal role in HCC development. Furthermore, shRNA KNTC1 (Lv-shKNTC1) was applied to infect BEL-7404 and SK-HEP-1 to identify roles of KNTC1 on HCC. Lv-shKNTC1 cells showed reduced proliferation ability, increased apoptosis and decreased migration ability. In vivo experiments suggested that xenografts grow significantly slower upon the silencing of KNTC1. Mechanistically, the protein levels of PIK3CA, p-Akt, CCND1, CDK6 are all down-regulated in Lv-KNTC1 SK-HEP-1 cells. Therefore, KNTC1 may affect the biological activity of HCC cells through PI3K/Akt signaling pathway.

Conclusions: In summary, the key finding of this report highlighted the significance of KNTC1 in tumor regression of HCC, demonstrating KNTC1 as an innovative target for adjuvant treatment of HCC.

Keywords: KNTC1, apoptosis, proliferation, PI3K/Akt, hepatocellular carcinoma

\section{Background}

The characteristics of high morbidity and mortality of liver cancer pose a great harm to human health[1]. Hepatocellular carcinoma (HCC) accounts for approximately $80 \%$ of all 
liver cancer types[2]. Liver cancer has the characteristics of rapid development, easy metastasis, severe infiltration, high malignant and poor prognosis[3]. Although with the development of medicine in recent years, its diagnostic and therapeutic management have been improved, including surgical resection, liver transplantation, radiofrequency ablation, chemoembolization and the multikinase inhibitor sorafenib[4], but the prognosis is still far from satisfying. Therefore, it is highly warranted to further explore the pathogenesis of HCC and identify novel therapeutic targets.

Cell division is regulated by a variety of proteins at all stages of cell cycle. The cell-cycle checkpoints are pivotal to genome maintenance and are specifically deregulated in cancer cells and some of them are considered as potential therapeutic targets[5]. Mitotic defects could lead to excess chromosomes and chromosomal instability (CIN) which was found in most cancer cells[6]. CIN was thought to be conducive to tumorigenesis, tumor progression, poor prognosis and the emergence of drug resistance[7]. Previous studies have reported that various proteins participating in the regulation of mitosis are abundant expressed in human tumor tissues, an amount of which are proved to be carcinogenic[8].

Kinetochores possesses complex multi-subunit structure that consist of more than 100 different proteins in human cells[9], which is imperative in maintaining the normal division of cell sister chromatid and maintaining chromosomal stability. Studies have indicated that kinetocyte proteins are vital in tumor. It was reported that KNTC2 was essential to maintain the normal process of cell mitosis [10] and was up-regulated in many tumor tissues, such as HCC, colon cancer, gastric cancer and pancreatic cancer[11-14]. Urata et al. identified that targeted knockdown of kinetochore protein D40 can prevent neoplasm cell progression[15]. Therefore, kinetochore proteins may be novel regulator in tumor development and progression, the further elucidating molecular biological mechanism of which may contribute to the accurate targeted therapy of tumors. Notably, hepatocellular carcinoma (HCC) cell lines performed high frequencies of aneuploidy, impaired mitotic checkpoints, and chromosomal instability[16-19]. Therefore, it is reasonable to deduce that the proteins involved in kinetochores are potential therapeutic targets of HCC.

Kinetochore-associated protein 1 (KNTC1), a kinetochore component in Rod-Zwilch-ZW10 (RZZ) complex, is not only highly conserved in evolution but also prominent for mitosis. It ensures the normal progress of spindle aggregation and chromosome segregation[20]. KNTC1, as a vital component of the mitotic checkpoint[20], may as a promising candidate for hepatocellular carcinoma treatment.

In this study, we found that KNTC1 was up-regulated in HCC tumor tissues, indicating that KNTC1 has a vital significance for the development of liver cancer. HCC cells with down-regulated expression of KNTC1 showed reduced proliferation ability, increased apoptosis and decreased migration ability. Consistently, xenografts formed by cells with KNTC1 knockdown grew relatively slower in vivo. Further molecular mechanistic studies revealed that the changes of biological activity of KNCT1 knockdown HCC cells may attribute to suppression of PI3K/Akt pathway. Therefore, perhaps KNTC1 serve as a meaningful candidate target for hepatocellular carcinoma gene silencing strategies.

\section{Methods}

Cell culture 
Hepatocellular carcinoma cell lines, including BEL-7404 and SK-HEP-1 (Shanghai Cell Biology Institute of Chinese Academy of Sciences, Shanghai, China) were incubated in DMEM (Thermo Fisher Scientific, MA, USA) and supplemented with 10\% FBS (Thermo Fisher Scientific, MA, USA) at an atmosphere of $5 \% \mathrm{CO}_{2}$ at $37^{\circ} \mathrm{C}$.

\section{Construction of KNTC1 knockdown cell model}

Short hairpin RNAs (shRNAs) targeted against KNTC1 mRNA (NM_014708) were generated, and scrambled control shRNA (shCtrl) was produced as negative control. The

sequences were summarized listed as below:
5'-CCGGTGGGGCATTCGTCTTGGTAAATTCAAGAGATTTACCAAGACGAATGCCCCATTTTTG-3' . After shRNA sequences were synthetized cloned into the BR-V108 (Shanghai Yibeirui Biomedical Science and Technology Co., Ltd) plasmid vector. Then, pHelper1.0, pHelper2.0 vectors, and the shRNA plasmids were co-transfected into 293T cells for 48 hours. The recombinant lentivirus was collected, which included LV-shKNTC1 (LV expressing KNTC1 shRNA) and LV-shCtrl (LV expressing scrambled shRNA), respectively. The hole - by dilution titer assay was applied to access infectious titer. BEL-7404 and SK-HEP-1 cells ( $3 \times$ $10^{5}$ cells/well) were incubated into 6 - well plates and then infected with LV-shKNTC1 or $\mathrm{LV}$-shCtrl $\left(1 \times 10^{8} \mathrm{TU} / \mathrm{ml}\right)$ at $70 \%$ to $80 \%$ confluences. The infection efficiency was estimated after 72 hours post infection.

\section{RNA extraction and qPCR}

The cells mRNA was obtained using TRIzol reagent (Life Technologies) according to the manufacturer's instructions. Subsequently, superscript first-strand synthesis system (Life Technologies) was applied to reverse transcription. Quantitative reverse transcription polymerase chain reaction (qPCR) (Applied Biosystems, Foster City, CA, USA) was used for relative quantification of KNTC1 gene expression. The relative expression values of KNTC1 were calculated using $2-\Delta \Delta \mathrm{Cp}$ method and housekeeping gene GAPDH was internal reference. The primers sequences were listed: KNTC1: 5'- TGAAACGCTGCTCCACAAC-3' and

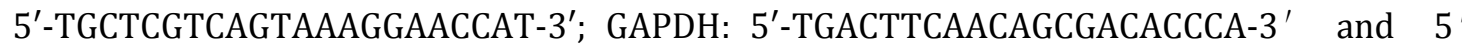
- CACCCTGTTGCTGTAGCCAAA - $3^{\prime}$.

\section{Immunohistochemical staining}

The specific anti- KNTC1 monoclonal antibody (biorbyt) was carried out to estimate the KNTC1 expression in 20 para-carcinoma tissues and 152 tumor tissues from different HCC patients by immunohistochemical staining. The main staining procedures were as follows: formalin-fixed paraffin-embedded 3- $\mu \mathrm{m}$ tissue microarray sections were subjected to deparaffinized and hydrated. $3 \% \mathrm{H}_{2} \mathrm{O}_{2}$ incubation for 10 min was used to eliminate endogenous peroxidase activity. After that incubation with anti- KNTC1 overnight at $4{ }^{\circ} \mathrm{C}$ and then with secondary antibody for $60 \mathrm{~min}$ at room temperature. DAB staining and hematoxylin counter staining were performed. The stained fields were photographed using microscopy.

\section{Western blot assay}

Total proteins extraction from BEL-7404 or SK-HEP-1 cells according to routine methods [21]. The proteins were separated by 8\% SDS-PAGE (Sigma-Aldrich, St. Louis, MO, USA) and transferred onto nitrocellulose membranes (GE Healthcare Life sciences, Pittsburgh, PA, USA). After the membranes were sealed with $5 \%$ non-fat milk at $37^{\circ} \mathrm{C}$ for 1 hour, primary antibodies (anti-KNTC1, biorbyt, 1:500; anti-GAPDH, Bioworld, 1:3000; 
anti-Akt, CST, 1:1000; anti-p-Akt, Bioss, 1:1000; anti-CCND1, CST, 1:2000; anti-CDK6, abcam, 1:1000; anti-PIK3CA, abcam, 1:1000) were incubated at $4^{\circ} \mathrm{C}$ overnight. The blots were detected by SuperSignal West Pico Chemiluminescent Substrate (Thermo Scientific, Rochford, IL, USA) after incubating with the secondary Goat Anti-Rabbit antibody (Beyotime, 1:3000) for 2 hours.

\section{MTT assay}

The infected cells were distributed into 96-well plate at 2000 cells per well and incubated to 4 hours with MTT ( $20 \mu \mathrm{L}$, Genview, Craigieburn, Victoria , Australia) at $5 \mathrm{mg} / \mathrm{ml}$ concentrations. $100 \mu \mathrm{L}$ of dimethyl sulfoxide were added to dissolve the methyl nitrogen crystals formed by living cells. After shaking for 2 to 5 minutes, the absorbance at $490 \mathrm{~nm}$ was detected by microplate reader (Tecan Infinite, Tecan $\mathrm{GmbH}$, Groedig, Austria). The detection time points were 1, 2, 3, 4, and 5 days after cultivation.

\section{Annexin V assay}

Fluorescein isothiocya-nate (FITC)-conjugated Annexin V (eBioscience; Thermo Fisher Scienti c, Inc.) was used for apoptosis detection. The infected cells $\left(2 \times 10^{5}\right.$ cells/well) were grew to about $70 \%$ coverage rate at 6 - well plate, harvested and then stained with (FITC)conjugated Annexin V. At least $5 \times 10^{5}$ cells were used to detect the FITC fluorescence signals via flow cytometry (Billerica, MA, USA).

\section{Colony formation assay}

The infected cells were seeded into 6-well plates at 400 to 1000 cells/well after transfection. After 2 weeks cultivation, cells were fixed with $4 \%$ paraformaldehyde for 30 min at room temperature and stained with $500 \mu \mathrm{L}$ Giemsa (Dingguo Changsheng, Beijing, China) for $20 \mathrm{~min}$. The total number of colonies were imaged and counted.

\section{Wound-healing assay}

$3 \times 10^{4}$ infected cells were cultured to at least $90 \%$ confluence at 96 - well plate. The scratch was formed at the central part of the 96 well plate by the scratch instrument. serum-free medium was used to rinse gently for 2-3 times. 0.5\% FBS/DMEM was added in to the wells. Wound widths of cell migration were obtained through microscope binding Image J software (NIH, Bethesda, MD, USA). The detection time points of BEL-7404 and SK-HEP-1 were at $0 \mathrm{~h}, 24 \mathrm{~h}, 50 \mathrm{~h}$ and $0 \mathrm{~h}, 8 \mathrm{~h}, 24 \mathrm{~h}$ respectively after scratching.

\section{Transwell migration assay}

Incubation $1 \times 10^{5}$ infected cells in 24 -well plate contained cell culture inserts (24-well, pore size $8 \mu \mathrm{m}$; Sigma-Aldrich Co.) and supplement with $100 \mu \mathrm{L}$ media without FBS. 600ul $30 \%$ FBS/DMEM was added into the lower chamber. After incubation for 48 hours (BEL-7404) or 16 hours (SK-HEP-1), noninvasive cells from the upper chamber were removed with a cotton swab. Invasive cells on the surface of the lower membrane were fixed with $5 \%$ crystal violet solution for 5 minutes and then photographed with an inverted microscope.

\section{Human apoptosis antibody array}

The expression levels of apoptosis related proteins involved in Lv-shKNTC1cells were detected by Human Apoptosis Antibody Array kit (abcam) according to the manufacturer's protocols after collection and lysis.

\section{Tumorigenesis and in vivo imaging in nude mice.}

Four-week-old BALB/c nude mice (15-20 g) were used and conducted strictly in accordance 
with the guidelines of the Animal Care and Use Committee of Shanghai Jiao Tong University. To evaluate SK-HEP-1 $\left(2 \times 10^{6}\right)$ cell proliferation in vivo, Lv-shKNTC1 or Lv-shCtrl were subcutaneously injected into armpit of right forelimb of the mice. Tumor growth curves were measured at 7, 12,14,16, 19 days after injection. Volume was accessed through the following formula: $\mathrm{V}=($ length $\times$ width 2 ) / 2. At 19 days following injection of HCC cells, 10 $\mu \mathrm{L} / \mathrm{g}$ D-Luciferin (Yeasen, Shanghai, China) were injected intraperitoneally into the mice. After 20 minutes, intraperitoneal injection of $0.7 \%$ pentobarbital sodium in anesthetized mice. Fluorescence results were observed using animal imaging system (PerkinElmer, Waltham, USA). All mice were sacrificed, and the tumors were incised, photographed and analyzed by IHC.

\section{Statistical analysis}

GraphPad Prism 6.0 software (GraphPadPrism 6.0 Software Inc., San Diego, CA, United States) were used in all statistical analyses. We used Mann-Whitney U test to analyze the differences between groups and the Spearman rank correlation test was used to confirm the correlations. The differences between the Lv-shKNTC1 group and Lv-shCtr group were analyzed by Student $t$ test. And differences between groups of shKNTC1 and shCtrl were assessed through one-way ANOVA. All data were showed as mean $\pm \mathrm{SD}$. P-values $<0.05$ were considered statistically significant.

\section{Results}

\section{KNTC1 expression is upregulated in HCC tissues}

To evaluate KNTC1 expression in HCC, it was detected in 152 HCC tissues and 20 para-carcinoma tissues by immunohistochemistry. Compared with para-carcinoma tissues, the expression of KNTC1 was significantly increased in HCC tissues (Table 1), representative pictures were shown in Figure 1. Consistently, the data mining of expression profiling of TCGA-LIHC database also showed the up-regulated expression of KNTC1 in HCC (Figure 2A). A statistical analysis of the relationship between KNTC1 expression and clinicopathological features in 152 patients. Depended on the median immunohistochemical score of patients, there were low expression group $(n=71)$ and high expression group $(n=81)$. It was declared that the expression level of KNTC1 was related to gender and tumor grade (Table 2). In addition, the Spearman rank correlation analysis demonstrated that the expression of KNTC1 gene was positively correlated with pathological grade and the expression of KNTC1 was higher in male patients (Table 3). More importantly, the Kaplan-Meier survival analysis of TCGA data indicated that patients with relatively high KNTC1 expression in tumor tissues generally suffer from poor prognosis (Figure 2B). All above results revealed the potential involvement of KNTC1 in HCC development and progression.

\section{KNTC1 expression is efficiently knocked-down by shRNA in HCC cells}

For constructing KNTC1 knockdown cell model, BEL-7404 and SK-HEP-1 cells infection Lv-shKNTC1 was KNTC1 downregulation and the infection shCtrl was negative control. More than $80 \%$ of green fluorescent cells were preliminarily observed in fluorescence imaging, indicating successful infection (Figure 3A, B). The qPCR analysis was used for further determination, showing that the mRNA expression of KNTC1 decreased sharply in the Lv-shKNTC1 groups with a knockdown efficiency of $96.4 \%$ in BEL-7404 cells and $75.1 \%$ in SK-HEP-1 cells $(\mathrm{P}<0.001$; Figure $3 \mathrm{C}, \mathrm{D})$. In addition, western blot analysis was carried out to 
confirm the depletion of KNTC1 in Lv-shKNTC1 groups (Figure 3E, F). All these findings indicated that Lv-shKNTC1 could efficiently knock down the expression of KNTC1 in BEL-7404 and SK-HEP-1 cells.

\section{Down-regulation of KNTC1 inhibits growth and induces apoptosis of HCC cells.}

The proliferation rate of HCC cells was determined by MTT assay and colony formation experiments. Compared with the group transfected with the Lv-shCtrl, BEL-7404 and SK-HEP-1 cells infected with Lv-shKNTC1 displayed significantly suppressed cell growth (Figure 4A, B) and inhibited ability of colony formation (Figure 4C-F). Subsequently, results of flow cytometry showed the apoptotic cell rate in Lv-shKNTC1 group had a sharp increase than that in $\mathrm{Lv}$-shCtrl group ( $\mathrm{P}<0.001$, Figure 4G-I). The above indicated KNTC1 may regulate development of HCC through influencing cell growth and apoptosis.

\section{Down-regulation of KNTC1 impedes migration of HCC cells.}

The effect of KNTC1 knockdown on cell migration of HCC was further studied. Wound-healing assay was carried out to estimate the role of KNTC1 on migration ability of HCC cells. KNTC1 knockdown significantly reduced the wound-closure capacity of Lv-shKNTC1 BEL-7404 cells (Figure 5A, B) and Lv-shKNTC1 SK-HEP-1 cells (Figure 5C, D). Correspondingly, the calculated migration rate of cells in Lv-shKNTC1 groups are decreased by $65 \%$ (50 h, BEL-7404, Figure 5B) and 24\% ( $24 \mathrm{~h}$, SK-HEP-1, Figure 5D) $(\mathrm{P}<0.001)$. Moreover, the role of KNTC1 in BEL-7404 and SK-HEP-1 cells migration was also tested by transwell assay. Lv-shKNTC1 groups showed a significant reduction in the average number of migrated cells (Figure 5E-J), indicating that migration ability was markedly decreased compared with Lv-shCtrl groups. Therefore, KNTC1 acts vital functions in the migration of HCC cells.

\section{Down-regulation of KNTC1 impedes tumor growth in vivo.}

In order to verify the conclusion of in vitro experiments, the tumorigenesis in nude mice were observed. Two groups of mice ( $\mathrm{n}=5$ per group) were injected with $\mathrm{Lv}$-shCtrl or Lv-shKNTC1 SK-HEP-1 cells. As shown in Figure 6A-D, compared with control group, tumors were detectable much earlier in Lv-shCtrl SK-HEP-1 cells injected group. And the growth rate and volume of tumor decreased significantly at each measurement point following injection with Lv-shKNTC1 SK-HEP-1 cells $(\mathrm{P}<0.05)$. Moreover, the immunohistochemistry analysis of Ki-67 (cell proliferation marker) was carried out to evaluate the proliferative activity of HCC cells in xenografts. As shown in Figure 6E, the number Ki-67 positive cells were remarkably less in Lv-shKNTC1 group in comparison with Lv-shCtrl group. These results demonstrated that silencing KNTC1 significantly inhibits the tumorigenicity of SK-HEP-1 cells in vivo.

\section{Anti-apoptosis proteins in Lv-shKNTC1 SK-HEP-1 cells were down-regulated.}

In order to interpret how KNTC1 affects cell apoptosis, we used human apoptosis antibody array kit to simultaneously detect the expression levels of 43 apoptosis related proteins (Figure 7A). As expected, a variety of anti-apoptosis proteins were down-regulated (Figure 7B, C and table 4), by which may infection of Lv-shKNTC1 induce cell apoptosis. The gray values of all the significantly changed proteins (Bcl-2, Bcl-w, HSP27, HSP60, IGF-I, IGF-II, Survivin, sTNF-R1, XIAP) are presented in Figure 7D.

\section{Down-regulation of KNTC1 induces suppression of the PI3K/AKT signaling pathway.}

The PI3K/Akt pathway is vital for proliferation, apoptosis and metastasis in many types 
of human cancers[22-24]. The intracellular lipid kinases family contains three different classes of enzymes, the most thoroughly studied of which is PI3K, consists of two unique subunits catalytic subunit (p110) and regulatory subunit (p85). PIK3CA, the p110 $\alpha$ catalytic subunit, is frequently mutated or overexpressed in many different human cancers[25-28]. Phosphatidylinositol - 3,4,5 - triphosphate (PIP3), a product catalyzed by PI3Ks, recruits and activates a wide range of downstream effectors. For them, Akt is considered the major downstream effector.

In our previous study, we found that the Lv-shKNTC1 knockdown HCCs showed increased apoptosis and decreased proliferation, colony formation, migration ability. In order to explore whether the changes of cell activity were caused by the change of PI3K / Akt pathway, we detected the expression of PIK3CA and its downstream effector (p-Akt). As expected, the protein level of PIK3CA and p-Akt were down-regulated in Lv-shKNTC1 SK-HEP-1 cells without change of Akt level (Figure 8), indicating that KNTC1 regulated the phosphorylation of Akt but not the expression level. The downstream targets of PI3K / Akt pathway were also detected. As shown in Figure 8, the expression of CCND1 and CDK6 was decreased in KNTC1 knockdown HCC cells compared with control group.

\section{Discussion}

Many tumors show a tendency to dysfunction during chromosome separation[29]. Kinetochore plays an important role in maintaining the normal division of cell sister chromatid during mitosis and maintaining chromosomal stability. Kinetochore acts as a bridge to ensure that the sister chromatids are properly connected to the spindle poles[30]. Numerous proteins localized to the kinetochores are involved in the accurately controlling of this process[31]. Many studies have reported that the expression of kinetochore genes in tumor tissues is up-regulated compared with normal control tissues[32-35]. Defects in kinetochore-associated proteins can lead to aneuploidy and chromosomal instability, which account for several diseases including HCC $[19,36]$. Therefore, the understanding of the role of kinetochore-associated proteins that regulates mitosis in HCC cells could benefit for exploring novel avenues and new therapies for patients with HCC. In the process of accurate chromosome separation, KNTC1 is a core protein, which mainly aggregates centromeric dynein, regulates sister chromatids to move to the extreme, and maintains mitotic mid-term checkpoints[37]. We demonstrated KNTC1 was highly expressed in liver cancer tissues in contrast with the para-carcinoma tissue. On the other hand, KNTC1 knockdown reduced proliferation ability, accelerated apoptosis and decreased migration ability of HCC cells. Therefore, as a kinetochore-associated protein, KNTC1 acts an oncogenic role in cell growth, migration and apoptosis of liver cancer.

Up-regulation of kinetochore genes was closely related to the biological behavior of tumors[38], and further study confirmed that overexpression of the kinetochore genes promotes aneuploidy and tumorigenesis[33, 39, 40]. Consistent with these studies, KNTC1 was newly found as an overexpressed kinetochore gene in HCC tissues. The results of clinical correlation study prompted that the expression of KNTC1 was notable correlation with tumor stage of HCC patients. In addition, abnormal mitosis, which can be caused by abnormal kinetochore protein, can lead to tumor metastasis, drug resistance, and poor treatment prognosis[41]. Altogether, KNTC1 may be an effective tumor regulator and a 
valuable therapeutic target in HCC.

In order to explore the biological role on HCC of KNTC1, we constructed lentivirus-short hairpin RNA KNTC1 to reduce KNTC1 expression in both BEL-7404 and SK-HEP-1 cells. Through MTT and clone formation assay, we confirmed that the proliferation capacity was significantly reduced in Lv-shKNTC1 HCC cells. Annexin V assay showed apoptosis was increased and the decreased migration ability was observed in wound-healing assay. And we also found that some anti-apoptosis proteins in Lv-shKNTC1 HCC cells were down-regulated, which resulted in the increase of apoptotic cells. In summary, these findings suggest that KNTC1 indeed have a tumorigenic effect in the formation and progression of hepatocellular carcinoma.

PI3K (phosphatidylinositol 3-kinase) and its downstream effective apparatus Akt (Protein Kinase B) are important regulatory factors in cell cycle, cell proliferation, cell metabolism and apoptosis[42]. Studies manifested that PI3K/Akt signaling pathway was also involved in mitosis. Akt is activated during mitosis, and inhibition of PI3K/Akt pathway impedes the cell cycle[43, 44]. Akt inhibition interferes with centrosomes separation and spindle formation, thus hindering mitotic processes[45]. Campa et al. reported that PI3K combined with CDK and other active factors can regulate the initiation and metaphase of mitosis and the localization of spindle[46]. Therefore, we supposed that KNTC1 may affect the biological function of HCC cells by regulating PI3K/Akt pathway. PI3K and Akt are major components of this pathway[47], which are necessary to induce the proliferation and differentiation of stem cells[48]. With the deepening of the understanding about the behavior diversity of hepatoma cells and complexity of microenvironment, PI3K/Akt pathway has become more and more prominent. Many drugs that target this pathway have been applied in clinical trials[49]. Mutations in gene PIK3CA which encodes the catalytic subunit p110 a can promote activation of the PI3K pathway[50], and p110 a mutation are very frequent in tumors[51]. P-Akt contributes to activation of Akt signaling pathway and its downstream genes[52]. During cell cycle, PI3K/Akt pathway regulates the expression of CyclinD1 (CCND1), which further activates CDK4 and CDK6, leading to protein substrate phosphorylation and cell proliferation[53,54]. To uncover possible molecular mechanisms, we detected protein levels of associated proteins, such as PIK3CA, p-Akt, CCND1 and CDK6. As expected, all the protein levels were down-regulated in Lv-shKNTC1 cells, indicating that KNTC1 knockdown reduces the biological activity of HCC cells by inhibiting the activity of PI3K/Akt pathway.

Taken together, our findings pointed out that silencing of KNTC1 can induce apoptosis and inhibit proliferation of hepatocellular carcinoma cells via suppressing PI3K/Akt pathway. According to our research, the combined application of KNTC1 gene knockdown and PI3K/Akt pathway inhibition may provide new strategies for the clinical treatment of liver cancer, improving the therapeutic effects and prognosis.

\section{Conclusions}

In summary, Our findings suggest that KNTC1 is essentially involved in the development and progression of HCC, indicating that KNTC1 may be as a new therapeutic target in HCC. 


\section{List of abbreviations}

KNTC1: Kinetochore associated 1

RZZ: Rod-Zwilch-ZW10

shRNA: short hairpin RNA

Lv-shKNTC1: short hairpin RNA (shRNA) KNTC1

HCC: Hepatocellular carcinoma

CIN: Chromosomes and chromosomal instability

\section{Declarations}

\section{Ethics approval and consent to participate}

All animal studies approved by the Animal Care and Use Committee of Shanghai Jiao Tong University and handled with care and euthanized humanely during the experiment.

\section{Consent for publication}

Not applicable.

\section{Availability of data and materials}

All data generated or analysed during this study are applicable.

\section{Competing interests}

The authors report no conflicts of interest in this work.

\section{Funding}

This study was supported by the National Natural Science Foundation of China (81972295) and the Subject of Biological Medicine of Science and Technology Commission of Shanghai Municipality (15411950404).

\section{Authors' contributions}

HT and JX performed the experiments and analyzed the data. XL and CP assisted with some experiments. ZZ and BS designed and supervised the experiments; HT and ZZ wrote the paper. All authors read and approved the final manuscript.

\section{Acknowledgements}

Not applicable.

\section{References}

1. Llovet JM, Zucman-Rossi J, Pikarsky E, Sangro B, Schwartz M, Sherman M, Gores G: Hepatocellular carcinoma. Nat Rev Dis Primers 2016, 2:16018.

2. Forner A, Reig M, Varela M, Burrel M, Feliu J, Briceno J, Sastre J, Marti-Bonmati L, Llovet JM, Bilbao Jl et al: [Diagnosis and treatment of hepatocellular carcinoma. Update consensus document from the AEEH, SEOM, SERAM, SERVEI and SETH]. Med Clin (Barc) 2016, 146(11):511 e511-511 e522.

3. Miao HL, Lei CJ, Qiu ZD, Liu ZK, Li R, Bao ST, Li MY: MicroRNA-520c-3p inhibits hepatocellular carcinoma cell proliferation and invasion through induction of cell apoptosis by targeting glypican-3. Hepatol Res 2014, 44(3):338-348.

4. EASL-EORTC clinical practice guidelines: management of hepatocellular carcinoma. J Hepatol 2012, 56(4):908-943.

5. Dominguez-Brauer C, Thu KL, Mason JM, Blaser H, Bray MR, Mak TW: Targeting Mitosis in Cancer: Emerging Strategies. Mol Cell 2015, 60(4):524-536. 
6. Rajagopalan H, Lengauer C: Aneuploidy and cancer. Nature 2004, 432(7015):338-341.

7. Burrell RA, McClelland SE, Endesfelder D, Groth P, Weller MC, Shaikh N, Domingo E, Kanu N, Dewhurst SM, Gronroos E et al: Replication stress links structural and numerical cancer chromosomal instability. Nature 2013, 494(7438):492-496.

8. Keen N, Taylor S: Aurora-kinase inhibitors as anticancer agents. Nat Rev Cancer 2004, 4(12):927-936.

9. de Wolf B, Kops G: Kinetochore Malfunction in Human Pathologies. Adv Exp Med Biol 2017, 1002:69-91.

10. Chen Y, Riley DJ, Chen PL, Lee WH: HEC, a novel nuclear protein rich in leucine heptad repeats specifically involved in mitosis. Mol Cell Biol 1997, 17(10):6049-6056.

11. Kaneko N, Miura K, Gu Z, Karasawa H, Ohnuma S, Sasaki H, Tsukamoto N, Yokoyama S, Yamamura A, Nagase $\mathrm{H}$ et al: siRNA-mediated knockdown against CDCA1 and KNTC2, both frequently overexpressed in colorectal and gastric cancers, suppresses cell proliferation and induces apoptosis. Biochem Biophys Res Commun 2009, 390(4):1235-1240.

12. $\mathrm{Qu} \mathrm{Y,} \mathrm{Li} \mathrm{J,} \mathrm{Cai} \mathrm{Q,} \mathrm{Liu} \mathrm{B:} \mathrm{Hec1/Ndc80} \mathrm{is} \mathrm{overexpressed} \mathrm{in} \mathrm{human} \mathrm{gastric} \mathrm{cancer} \mathrm{and} \mathrm{regulates}$ cell growth. J Gastroenterol 2014, 49(3):408-418.

13. Meng QC, Wang HC, Song ZL, Shan ZZ, Yuan Z, Zheng Q, Huang XY: Overexpression of NDC80 is correlated with prognosis of pancreatic cancer and regulates cell proliferation. Am $J$ Cancer Res 2015, 5(5):1730-1740.

14. Huang LY, Chang CC, Lee YS, Huang JJ, Chuang SH, Chang JM, Kao KJ, Lau GM, Tsai PY, Liu CW et al: Inhibition of Hec1 as a novel approach for treatment of primary liver cancer. Cancer Chemother Pharmacol 2014, 74(3):511-520.

15. Urata YN, Takeshita F, Tanaka H, Ochiya T, Takimoto M: Targeted Knockdown of the Kinetochore Protein D40/Knl-1 Inhibits Human Cancer in a p53 Status-Independent Manner. Sci Rep 2015, 5:13676.

16. Saeki A, Tamura S, Ito N, Kiso S, Matsuda Y, Yabuuchi I, Kawata S, Matsuzawa Y: Frequent impairment of the spindle assembly checkpoint in hepatocellular carcinoma. Cancer 2002, 94(7):2047-2054.

17. Attallah AM, Tabll AA, Salem SF, El-Sadany M, Ibrahim TA, Osman S, El-Dosoky IM: DNA ploidy of liver biopsies from patients with liver cirrhosis and hepatocellular carcinoma: a flow cytometric analysis. Cancer Lett 1999, 142(1):65-69.

18. Huang SF, Hsu HC, Fletcher JA: Investigation of chromosomal aberrations in hepatocellular carcinoma by fluorescence in situ hybridization. Cancer Genet Cytogenet 1999, 111(1):21-27.

19. Jeong SJ, Shin HJ, Kim SJ, Ha GH, Cho BI, Baek KH, Kim CM, Lee CW: Transcriptional abnormality of the hsMAD2 mitotic checkpoint gene is a potential link to hepatocellular carcinogenesis. Cancer Res 2004, 64(23):8666-8673.

20. Chan GK, Jablonski SA, Starr DA, Goldberg ML, Yen TJ: Human Zw10 and ROD are mitotic checkpoint proteins that bind to kinetochores. Nat Cell Biol 2000, 2(12):944-947.

21. Wang KK, Posner A, Hajimohammadreza I: Total protein extraction from cultured cells for use in electrophoresis and western blotting. Biotechniques 1996, 20(4):662-668.

22. Sun Y, Tian H, Wang L: Effects of PTEN on the proliferation and apoptosis of colorectal cancer cells via the phosphoinositol-3-kinase/Akt pathway. Oncol Rep 2015, 33(4):1828-1836. 
23. Nakatsuka T, Tateishi K, Kudo Y, Yamamoto K, Nakagawa H, Fujiwara H, Takahashi R, Miyabayashi K, Asaoka Y, Tanaka $Y$ et al: Impact of histone demethylase KDM3A-dependent AP-1 transactivity on hepatotumorigenesis induced by PI3K activation. Oncogene 2017, 36(45):6262-6271.

24. Ge JC, Wang YX, Chen ZB, Chen DF: Integrin alpha 7 correlates with poor clinical outcomes, and it regulates cell proliferation, apoptosis and stemness via PTK2-PI3K-Akt signaling pathway in hepatocellular carcinoma. Cell Signal 2020, 66:109465.

25. Cantley LC: The phosphoinositide 3-kinase pathway. Science 2002, 296(5573):1655-1657.

26. Jung $\mathrm{K}$, Kang H, Mehra R: Targeting phosphoinositide 3-kinase (PI3K) in head and neck squamous cell carcinoma (HNSCC). Cancers Head Neck 2018, 3:3.

27. Akagi I, Miyashita M, Makino H, Nomura T, Hagiwara N, Takahashi K, Cho K, Mishima T, Ishibashi $\mathrm{O}$, Ushijima $\mathrm{T}$ et al: Overexpression of PIK3CA is associated with lymph node metastasis in esophageal squamous cell carcinoma. Int J Oncol 2009, 34(3):767-775.

28. Wang C, Che L, Hu J, Zhang S, Jiang L, Latte G, Demartis MI, Tao J, Gui B, Pilo MG et al: Activated mutant forms of PIK3CA cooperate with RasV12 or c-Met to induce liver tumour formation in mice via AKT2/mTORC1 cascade. Liver Int 2016, 36(8):1176-1186.

29. Thompson SL, Bakhoum SF, Compton DA: Mechanisms of chromosomal instability. Curr Biol 2010, 20(6):R285-295

30. Cheeseman IM, Desai A: Molecular architecture of the kinetochore-microtubule interface. Nat Rev Mol Cell Biol 2008, 9(1):33-46.

31. Cleveland DW, Mao Y, Sullivan KF: Centromeres and kinetochores: from epigenetics to mitotic checkpoint signaling. Cell 2003, 112(4):407-421.

32. Ryan SD, Britigan EM, Zasadil LM, Witte K, Audhya A, Roopra A, Weaver BA: Up-regulation of the mitotic checkpoint component Mad1 causes chromosomal instability and resistance to microtubule poisons. Proc Natl Acad Sci U S A 2012, 109(33):E2205-2214.

33. Sotillo R, Hernando E, Diaz-Rodriguez E, Teruya-Feldstein J, Cordon-Cardo C, Lowe SW, Benezra R: Mad2 overexpression promotes aneuploidy and tumorigenesis in mice. Cancer Cell 2007, 11(1):9-23.

34. Grabsch H, Takeno S, Parsons WJ, Pomjanski N, Boecking A, Gabbert HE, Mueller W: Overexpression of the mitotic checkpoint genes BUB1, BUBR1, and BUB3 in gastric cancer--association with tumour cell proliferation. J Pathol 2003, 200(1):16-22.

35. Mondal G, Sengupta S, Panda CK, Gollin SM, Saunders WS, Roychoudhury S: Overexpression of Cdc20 leads to impairment of the spindle assembly checkpoint and aneuploidization in oral cancer. Carcinogenesis 2007, 28(1):81-92.

36. Orr B, Godek KM, Compton D: Aneuploidy. Curr Biol 2015, 25(13):R538-542.

37. Scaerou F, Starr DA, Piano F, Papoulas O, Karess RE, Goldberg ML: The ZW10 and Rough Deal checkpoint proteins function together in a large, evolutionarily conserved complex targeted to the kinetochore. J Cell Sci 2001, 114(Pt 17):3103-3114.

38. Yuen KW, Montpetit B, Hieter P: The kinetochore and cancer: what's the connection? Curr Opin Cell Biol 2005, 17(6):576-582.

39. Diaz-Rodriguez E, Sotillo R, Schvartzman JM, Benezra R: Hec1 overexpression hyperactivates the mitotic checkpoint and induces tumor formation in vivo. Proc Natl Acad Sci U S A 2008, 105(43):16719-16724.

40. Ricke RM, Jeganathan KB, van Deursen JM: Bub1 overexpression induces aneuploidy and 
tumor formation through Aurora B kinase hyperactivation. J Cell Biol 2011, 193(6):1049-1064.

41. Thompson SL, Compton DA: Chromosomes and cancer cells. Chromosome Res 2011, 19(3):433-444.

42. Vivanco I, Sawyers CL: The phosphatidylinositol 3-Kinase AKT pathway in human cancer. Nat Rev Cancer 2002, 2(7):489-501.

43. Roberts EC, Shapiro PS, Nahreini TS, Pages G, Pouyssegur J, Ahn NG: Distinct cell cycle timing requirements for extracellular signal-regulated kinase and phosphoinositide 3-kinase signaling pathways in somatic cell mitosis. Mol Cell Biol 2002, 22(20):7226-7241.

44. Shtivelman E, Sussman J, Stokoe D: A role for PI 3-kinase and PKB activity in the G2/M phase of the cell cycle. Curr Biol 2002, 12(11):919-924.

45. Liu X, Shi Y, Woods KW, Hessler P, Kroeger P, Wilsbacher J, Wang J, Wang JY, Li C, Li Q et al: Akt inhibitor a-443654 interferes with mitotic progression by regulating aurora a kinase expression. Neoplasia 2008, 10(8):828-837.

46. Campa CC, Martini M, De Santis MC, Hirsch E: How PI3K-derived lipids control cell division. Front Cell Dev Biol 2015, 3:61.

47. Brazil DP, Hemmings BA: Ten years of protein kinase B signalling: a hard Akt to follow. Trends Biochem Sci 2001, 26(11):657-664.

48. Downward J: Mechanisms and consequences of activation of protein kinase B/Akt. Curr Opin Cell Biol 1998, 10(2):262-267.

49. Spangle JM, Roberts TM, Zhao JJ: The emerging role of PI3K/AKT-mediated epigenetic regulation in cancer. Biochim Biophys Acta Rev Cancer 2017, 1868(1):123-131.

50. Ikenoue T, Kanai F, Hikiba Y, Obata T, Tanaka Y, Imamura J, Ohta M, Jazag A, Guleng B, Tateishi $\mathrm{K}$ et al: Functional analysis of PIK3CA gene mutations in human colorectal cancer. Cancer Res 2005, 65(11):4562-4567.

51. Fruman DA, Rommel C: PI3K and cancer: lessons, challenges and opportunities. Nat Rev Drug Discov 2014, 13(2):140-156.

52. Shan ZZ, Chen PN, Wang F, Wang J, Fan QX: Expression of P-EGFR and P-Akt protein in esophageal squamous cell carcinoma and its prognosis. Oncol Lett 2017, 14(3):2859-2863.

53. He YY, Council SE, Feng L, Chignell CF: UVA-induced cell cycle progression is mediated by a disintegrin and metalloprotease/epidermal growth factor receptor/AKT/Cyclin D1 pathways in keratinocytes. Cancer Res 2008, 68(10):3752-3758.

54. Yang X, Gong Y, He Q, Licht JD, Liaw L, Friesel RE: Loss of Spry1 attenuates vascular smooth muscle proliferation by impairing mitogen-mediated changes in cell cycle regulatory circuits. J Cell Biochem 2018, 119(4):3267-3279.

\section{Figure legend}

Fig.1 Immunohistochemistry of KNTC1 expression in para-carcinoma tissue (a) and liver cancer tissue (b). (DAB staining, hematoxylin counterstain, magnification: $400 \mathrm{x}$ )

Fig.2 The data mining of TCGA database. a mRNA expression of KNTC1 in HCC was up-regulated. b The Kaplan-Meier survival analysis of TCGA data. 
Fig.3 Infection efficiency and gene knockdown efficiency of Lv-shKNTC1 in BEL-7404 and SK-HEP-1 cells. (a, b) Bright field and GFP observation of BEL-7404 and SK-HEP-1 cells after infecting Lv-shCtrl or Lv-shKNTC1. Magnification, x200. The results indicated that Lv-shshKNTC1 and Lv-shCtrl effectively infected BEL-7404 and SK-HEP-1 cells. The green fluorescence was observed in over 80\% cells. (c, d) mRNA expression levels of KNTC1 were significantly decreased in the Lv-shKNTC1 groups, with a knockdown efficiency of $96.4 \%$ in BEL-7404 cells and $75.1 \%$ in SK-HEP-1 cells $(\mathrm{P}<0.001)$ compared with the Lv-shCtrl groups. (e, f)Western blot of KNTC1 in Lv-shKNTC1 or Lv-shCtrl infected BEL-7404 and SK-HEP-1 cells. Protein expression of KNTC1 in Lv-shKNTC1 groups was significantly decreased compared with the control groups. ${ }^{* * *} \mathrm{P}<0.001$, Ctrl, control; KNTC1, kinetochore associated 1; Lv, lentivirus; sh, short hairpin RNA.

Fig.4 Effects of KNTC1 gene knockdown on growth and apoptosis of BEL-7404 and SK-HEP-1 cells. (a, b) MTT assay was performed. The absorbance at $490 \mathrm{~nm}$ was detected and compared. The number of active BEL-7404 and SK-HEP-1 cells was significantly reduced in Lv-shKNTC1 groups. (c, d) Colony formation assay of BEL-7404 cells. The number of clones of Lv-shKNTC1 BEL-7404 cells was obviously decreased compared with the Lv-shCtrl group $(\mathrm{P}<0.001)$. (e, f) Colony formation assay of SK-HEP-1 cells. The number of clones of Lv-shKNTC1 SK-HEP-1 cells was significantly reduced compared with the Lv-shCtrl group $(\mathrm{P}<0.01)$. Apoptotic cells were stained with Annexin V-APC and measured with flow cytometry (g, h) Images and statistical graph of apoptosis level in Lv-shKNTC1 and Lv-shCtrl BEL-7404 cells. The results showed that the percentage of apoptotic cells was significantly increased in the Lv-shKNTC1 BEL-7404 cells $(\mathrm{P}<0.001)$. (i, j) Images and statistical graph of apoptosis level in Lv-shKNTC1 and Lv-shCtrl SK-HEP-1 cells. The proportion of apoptotic cells was significantly increased in the Lv-shKNTC1 SK-HEP-1 cells $(\mathrm{P}<0.001)$.

Fig.5 Effects of KNTC1 gene knockdown on the migration of HCC cells. (a, b) Images and statistical graph of migration ability of Lv-shKNTC1 and Lv-shCtrl BEL-7404 cells. (c, d) Images and statistical graph of migration ability of Lv-shKNTC1 and Lv-shCtrl SK-HEP-1 cells. The relative widths of the wound gaps were measured by Image J software. All data are expressed as means $\pm \mathrm{SD} .{ }^{* *} \mathrm{P}<0.01$. ${ }^{* * *} \mathrm{P}<0.001$, compared with the Lv-shCtrl groups. $\mathrm{e}$ Transwell assay was performed to measure cell migration capability of BEL-7404 cells. (f, g) Statistical graph of transwell assay result of BEL-7404 cells. h Transwell assay was performed to measure cell migration capability of SK-HEP-1 cells. (i, j) Statistical graph of transwell assay result of SK-HEP-1 cells. The number of cells was counted and imaged with an inverted microscope (magnification: $200 \times$ ). ${ }^{* * *} \mathrm{P}<0.001$, compared with the Lv-shctrl groups. All data are expressed as means \pm SD.

Fig.6 Effects of KNTC1 knockdown on tumorigenesis in nude mice in vivo. a Images of nude mice and their corresponding xenograft tumors. b Fluorescence images of xenograft tumors from the Lv-shKNTC1 or Lv-shCtrl SK-HEP-1 cells. c Volume of subcutaneous xenograft tumors at 7, 12, 14, 16, 18 days after injected Lv-shKNTC1 or Lv-shCtrl SK-HEP-1 cells. d Statistical graph of total fluorescence intensity. ${ }^{* * *} \mathrm{P}<0.001$, compared with the Lv-shctrl 
groups. e HE staining of xenograft tumors and immunohistochemistry of Ki67.

Fig.7 Effects of KNTC1 gene knockdown on apoptosis related genes in SK-HEP-1 cells. a The original result of chemiluminescence. b A schematic diagram of the chemiluminescence results. $\mathbf{c}$ Fold changes of all the apoptosis related proteins. d Statistical map of all proteins with expression changes and statistical significance. ${ }^{*} \mathrm{P}<0.05$, compared with the Lv-shctrl groups.

Fig.8 Effect of KNTC1 knockdown of PI3K/Akt signaling pathway. Western blot shows that the protein levels of PIK3CA, p-Akt, CCND1, CDK6 are all down-regulated in Lv-KNTC1 group. 